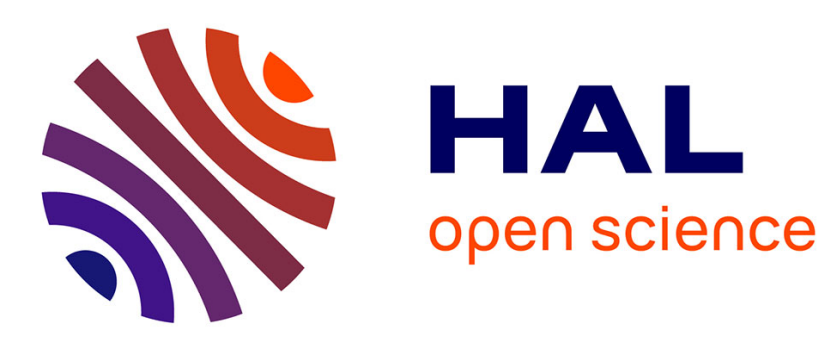

\title{
Spatially-variant morpho-Hessian filter: Efficient implementation and application
}

Olena Tankyevych, Hugues Talbot, Petr Dokládal, Nicolas Passat

\section{To cite this version:}

Olena Tankyevych, Hugues Talbot, Petr Dokládal, Nicolas Passat. Spatially-variant morpho-Hessian filter: Efficient implementation and application. International Symposium on Mathematical Morphology (ISMM), 2009, Groningen, Netherlands. pp.137-148, 10.1007/978-3-642-03613-2_13 . hal00622432

\section{HAL Id: hal-00622432 \\ https://hal.science/hal-00622432}

Submitted on 25 Sep 2013

HAL is a multi-disciplinary open access archive for the deposit and dissemination of scientific research documents, whether they are published or not. The documents may come from teaching and research institutions in France or abroad, or from public or private research centers.
L'archive ouverte pluridisciplinaire HAL, est destinée au dépôt et à la diffusion de documents scientifiques de niveau recherche, publiés ou non, émanant des établissements d'enseignement et de recherche français ou étrangers, des laboratoires publics ou privés. 


\title{
Spatially-variant morpho-Hessian filter: efficient implementation and application
}

\author{
Olena Tankyevych ${ }^{1}$, Hugues Talbot ${ }^{1}$, Petr Dokladál $^{2}$, and Nicolas Passat ${ }^{3}$ \\ 1 Université Paris-Est, Département d'Informatique Gaspard-Monge, \\ Équipe A3SI, ESIEE Paris, Cité Descartes, BP 99, FR-93162 Noisy-le-Grand Cedex, \\ France \\ \{tankyevo, talboth\}@esiee.fr, \\ 2 Centre de Morphologie Mathématique, Mines-Paristech, 35 rue St Honoré, F-77300 \\ Fontainebeau, France \\ petr.dokladal@mines-paristech.fr, \\ 3 Université de Strasbourg, LSIIT UMR 7005, Strasbourg, France \\ passat@unistra.fr
}

\begin{abstract}
Elongated objects are more difficult to filter than more isotropic ones because they locally comprise fewer pixels. For thin linear objects, this problem is compounded because there is only a restricted set of directions that can be used for filtering, and finding this local direction is not a simple problem. In addition, disconnections can easily appear due to noise. In this paper we tackle both issues by combining a linear filter for direction finding and a morphological one for filtering. More specifically, we use the eigen-analysis of the Hessian for detecting thin, linear objects, and a spatially-variant opening or closing for their enhancement and reconnection. We discuss the theory of spatially-variant morphological filters and present an efficient algorithm. The resulting spatially-variant morphological filter is shown to successfully enhance directions in 2D and 3D examples illustrated with a brain blood vessel segmentation problem.
\end{abstract}

Keywords: spatially-variant morphology.

\section{Introduction}

In this paper, we define thin objects in image as semantically consistent objects that exhibit at least one dimension much smaller than the others. We focus particularly on elongated thin objects, locally curve- or line-like, e.g. fibres, hair, strings, or blood vessels.

It is generally difficult to filter such objects. Indeed, classical filters in the literature assume extended objects (median, averaging, linear convolutions, morphological filters with standard structuring elements) [1,2]. Those that do not make this assumption still filter only in areas of low gradient in a region of interest [3-5]. Elongated objects may in fact present no part with a suitable low gradient, due to both noise and object edges, as in Figure 1. For elongated objects, in the literature, it is often assumed that one dimension is long, everywhere 


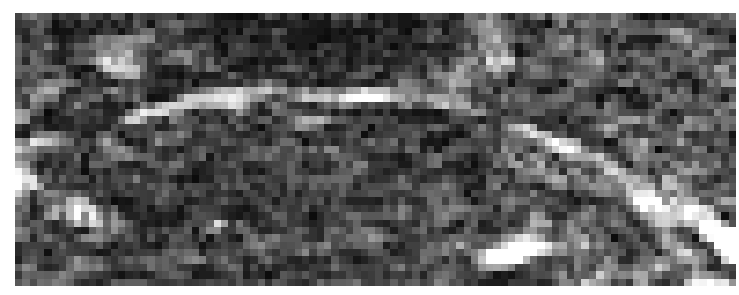

Fig. 1. A thin, noisy brain blood vessel. There is no low-gradient zone in this object, disconnections are due to noise.

tangent to the object. Filtering can be applied along this direction without necessarily destroying the object entirely. Within the morphology framework, one classically use families of segments as structuring elements (SEs), selecting the one best matching each object [6]. This, however, requires directional sampling, which may be prohibitive in 3D. Alternatively, path-based families of structuring elements can be used instead with no sampling required, but with similar complexities and running times $[7,8]$. Finally, in [9], curvature evaluation was also used in 2D in conjunction with mathematical morphology operators.

Linear (in the mathematical sense) filtering methods have also been proposed. In the case of the scale-space framework, which should be suitable to this problem, edge and ridge detection methods were proposed $[10,11]$ utilising the Hessian or the structure tensor [12,13]. As an extension of these methods, anisotropic diffusion is often proposed for filtering, using the tensorial information for diffusing only within the object. In practice diffusion may fail if the object is very thin [14].

Elongated objects have a tendency to be sensitive to noise, often implying disconnection along the object, as is readily apparent in Figure 1. Segmentation of this kind of objects is often reliant on connection being maintained throughout the object. Diffusion is often not well suited for this task $[15,14]$ as it will become isotropic outside the oriented objects.

In order to achieve reconnection, disconnected sets can be matched [16], but this is an ill-posed problem. Minimal path methods have been proposed to achieve reconnection $[17,18]$, but the problem of specifying endpoints can be difficult to solve.

Recently, two similar 2D approaches were proposed combining the analysis of orientation from either the Hessian or the structure tensor, and using spatiallyvariant (SV) morphology to bridge the disconnection gaps $[19,20]$. This is a productive approach, as long as the resulting filter is indeed a morphological one, and directions are well estimated.

Spatially-variant mathematical morphology (SVMM) was introduced in [21] for binary sets using structuring functions. In [22] it was used for adaptive filtering on grey-level and colour images. An efficient algorithm for variable rectangles was proposed in [23], finally, SVMM was studied from the theoretical point of view in $[24-26]$. 
In this article, in the first section we expose a simple version of the theory of SVMM together with an efficient algorithm. In the second section we briefly introduce multi-scale Hessian analysis for the study of local directions. In the third section we present applications using both concepts combined.

\section{Spatially-Variant Morphological Filtering}

The theory of SVMM and corresponding algorithms are formulated with the purpose of filtering an image differently at various positions. In the case of elongated objects, we wish to discover their local orientation and filter them locally along this direction, for instance, with an oriented segment.

This presents a challenge. Traditionally one could use a supremum of openings or an infimum of closings with a family of segments or paths as SEs, but the range of this kind of filters is limited. For instance, there is no known way to produce in this manner a morphological equivalent to an edge-enhancing, orientation-driven, inverse diffusion filter as in [12].

In this section we present the theory upon which, the filter presented in section 4 relies on.

\subsection{Adjunction and spatially-variant morphology}

First, we note that it is not useful to establish a distinction between binary and grey-level SVMM. Indeed, SVMM can be described on an arbitrary lattice in which SEs are available. In general, one can construct a simple SV morphological operator by computing a max or min filter using an SE that is not the same everywhere in the image. For instance, one can use parametrized disks or oriented segments, and vary respectively the diameter or the orientation according to a scalar field. Erosions and dilations with these SEs pose no problem by themselves. However, for filtering, openings and closings are the more interesting operators, but adjunct respective dilations and erosions are not trivially computed, as we shall see shortly..

Note that in general even though one can perform a kind of pseudo-opening or closing operation in each separate SE (by combining a max and min filter in the same window), the result of such an operator is not guaranteed to be idempotent due to the change of homothecy or rotation, and would furthermore be dependent on the order of operations. Consequently, it will not be a morphological filter [2].

In Figure 2, the concept of adjunction is illustrated in the case of SE-based operators. In Figure 2(a) we have the translation-invariant (TI) case of a $2 \times 1$ horizontal line segment. In the general case, the transpose of a SE $B$, noted $\check{B}$ is

$$
\check{B}(x)=\{y \mid x \in B(y)\},
$$

where $x$ and $y$ are points, and $B(y)$ is the potentially spatially-variant structuring element originating at point $y$. For a TI operator as in Figure 2(a), we observe that the general definition collapses to the usual one, i.e, $\check{B}=-B$, the symmetric 
of $B$ with respect to the origin. However, for the SV case this is not true. In Figure 2(b), we use the same SE, but with varying orientation (arrows denote directions). In Fig. 2(b), for the indicated pixel, the transpose of the SE is not a segment.

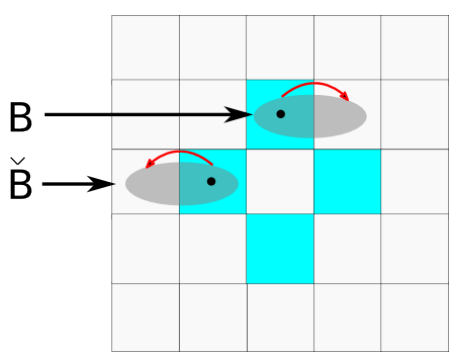

(a) TI case.

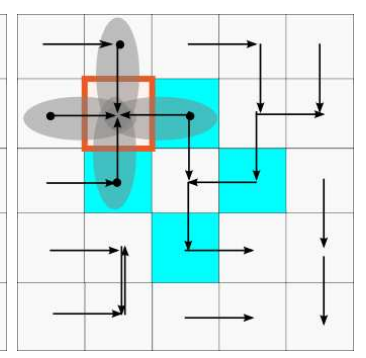

(b) SV case.

Fig. 2. Adjunct structuring elements with explicit formulation for $\check{B}$ for the TI case but not the SV one (as for the highlighted pixel).

Computing openings and closings with SV erosions and dilations requires the transpose to be computed everywhere, which can be computationally expensive requiring an exhaustive search. This becomes prohibitive for large, $n \mathrm{D}$ data $(n \geq 2)$. In the next section we present a way to decrease the computation complexity.

\subsection{Adjunct by conditional propagation}

In the following, we describe the SV dilation $\delta$ and its adjunct erosion $\varepsilon$ both using spatially-variant SEs .

Let $\mathcal{L}$ be a family of functions, or images, defined as a mapping from the support $D$ to the set of values $V$. Let $\delta, \varepsilon: \mathcal{L} \rightarrow \mathcal{L}$ be a dilation and an erosion of $f \in \mathcal{L}$ given by:

$$
\begin{aligned}
& {\left[\delta_{B}(f)\right](x)=\left[\bigvee_{b \in B(x)} f_{b}\right](x)} \\
& {\left[\varepsilon_{\check{B}}(f)\right](x)=\left[\bigwedge_{b \in \breve{B}(x)} f_{b}\right](x),}
\end{aligned}
$$

where $f_{b}$ denotes the translation of $f$ by $b$ computed as $f_{b}(x)=f(x-b)$. $B$ stands for the structuring element. In standard mathematical morphology, $B$ is translation invariant, defined as $B \subset D$.

In the context of SVMM, $B$ is often denoted as structuring function and is defined as $B: D \rightarrow \mathcal{P}(D), \mathcal{P}$ being the collection of all subsets of $D$. As above, $B(x)$ denotes the structuring element $B$ originating at point $x$. 
The transpose of $B$, denoted $\check{B}$, is defined in Eq. 1 and used in Eq. 3. This formulation of $\check{B}$ can be computationally costly. It can a priori be of arbitrary extent depending on the $B$ family, which becomes problematic for forming filters based on adjunctions of dilations and erosions in order to make a closing $\varphi_{B}$ or an opening $\gamma_{B}$ :

$$
\varphi_{B}(f)=\varepsilon_{\breve{B}}\left(\delta_{B}(f)\right) ; \quad \gamma_{B}(f)=\delta_{B}\left(\varepsilon_{\breve{B}}(f)\right) .
$$

Note that there also exists a complementary, SE-based SV erosion $\varepsilon_{B}(f)=$ $-\delta_{B}(-f)$ and its adjunct $\delta_{\check{B}}(f)$, and that in general $\varepsilon_{B}$ and $\varepsilon_{\check{B}}$ differ.

In the following we propose an implementation of the adjunct operator identical in complexity to the normal one.

Implementation The inf/sup-of-functions in Eqs. 2 and 3 are usually computed sequentially in raster scan order according to this scheme:

$$
\begin{aligned}
& {\left[\delta_{B}(f)\right](x)=\max _{b \in B(x)} f(x-b)} \\
& {\left[\varepsilon_{\breve{B}}(f)\right](x)=\min _{b \in \breve{B}(x)} f(x-b)=\min _{y \mid x \in B(y)} f(x-y)}
\end{aligned}
$$

The dilation of Eq. 5 is computed in $\mathcal{O}(M N)$, where $N=\operatorname{Card}(D)$, and $M=\operatorname{Card}(B(x))$. In the following erosion, from Eq. 6, given some $x$, the set $\{y \mid x \in B(y)\}$ is a priori unknown and is computed in $\mathcal{O}\left(N^{2}\right)$ by exhaustive search. However, relaxing the sequential order of computing, the adjunct erosion of Eq. 6 can be computed more efficiently, as shown below.

Theorem 1 (Conditional propagation for the adjunct erosion). Assume $\varepsilon_{\check{B}}(f)$, i.e. the output, originally set everywhere equal to $f$. One can sequentially read the input $f$ at each point $x$. Considering the structuring element $B(x)$ of origin $x$, for all elements $y$ of $B(x)$, we update the output value $\left[\varepsilon_{\check{B}}(f)\right](y)$ by taking the min operator between the current input value at $x$ and the current output value at $y$.

Proof. It is easy to show that both ways of computing the adjunct are equivalent. As we scan the input image at $x$ and update the value in the output image at $y$, we are indeed computing a min operator between all the origins of $B(x)$ such that $B(x)$ intersects $y$. So, once the whole image has been scanned, if $B(x)$ intersects $y$, then $x$ is in $\check{B}(y)$ and vice-versa.

This theorem is illustrated in Figure 3. In the Fig. 3(a) the original image is illustrated with the directions at each pixel as arrows. The structuring element $B$ is originating at the selected pixel $x$ for a SE-based dilation $\delta_{B}$. The result of this dilation is shown in the Fig. 3(b). In the same figure the adjunct structuring elements $\check{B}$ are pointing at the selected pixel $y$ for the efficient adjunct erosion. This operation is performed with the minimal value updated everywhere along $\check{B}$ as it spans the image, the result can be observed in the Fig. 3(c). 


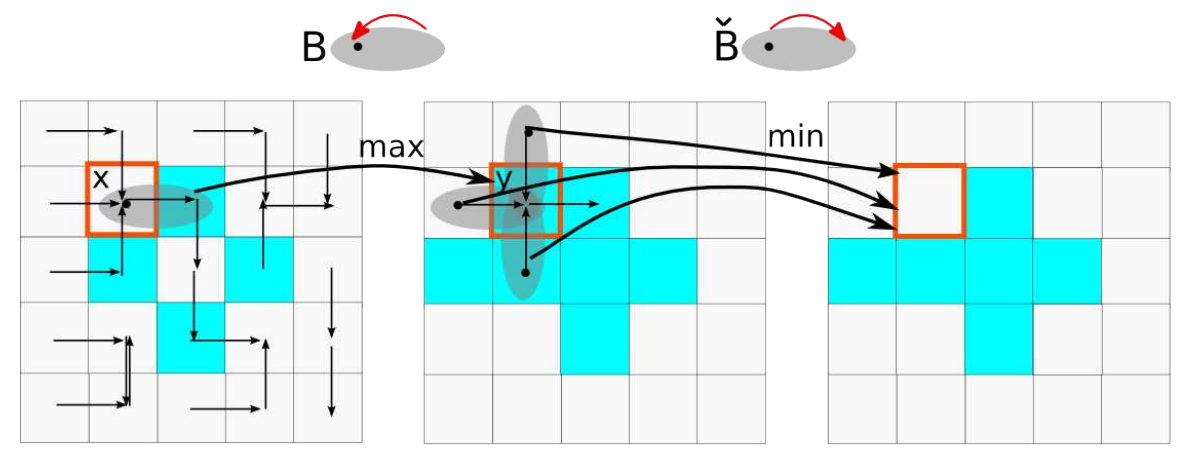

(a) Original image.

(b) SE-based dilation and its SE B. (c) The adjunct erosion and its SE $\check{\text { B }}$

Fig. 3. Efficient SV closing (arrows at each pixel denote directions, thick long arrows indicate the operation on the selected pixel).

This theorem leads to Algorithm 1 based on a conditional propagation of the value at the origin of each $B(x)$. In this algorithm, the for loop (lines 4-5) computes sequentially for all $x \in D$, the dilation $\delta_{B}(f)$ implemented by definition of Eq. 5. Every $\delta_{B}(f)$ is then stored in $d$ (previously initialized (line 3$)$ ). Notice that there is no need to store the entire image $\delta_{B}(f)$. The erosion is implemented by a conditional propagation (lines 6-7).

The result $\varphi_{B}(f)$ is available as soon as the raster scan of the input image ends (lines 2-7). The overall computational complexity is $\mathcal{O}\left(\sum_{x \in D} \operatorname{Card}(B(x))\right)$. If $B$ is of constant size, this reduces to $\mathcal{O}(M N)$, where $N=\operatorname{Card}(D)$, and $M=\operatorname{Card}(B)$. The SV opening can be computed analogously.

Notes (1) In the illustrated algorithm the closing starts with the SE-based dilation, but it would be possible to start with the complementary, adjunct dilation $\delta_{\breve{B}}$, followed by the SE-based erosion $\varepsilon_{B}$. The two results differ in general. (2) Forming an opening starting with the adjunct erosion requires intermediary storage. (3) SE can be of any shape, not just a line segment.

In the following section we propose a way to derive an orientation tensor field.

\section{Multiscale Hessian analysis}

For a real, twice derivable function $L\left(x_{1}, \ldots, x_{i}, \ldots, x_{n}\right)$ on a support $D$, the Hessian operator is the order- 2 tensor of the second derivatives

$$
H=\left(\frac{\partial^{2} L}{\partial x_{i} \partial x_{j}}\right)_{1 \leq i, j \leq n} .
$$

If the second derivatives are continuous, $H$ is symmetric semi-definite. In computer vision, the derivative operator $\partial$ is not well-defined because of digitization, 


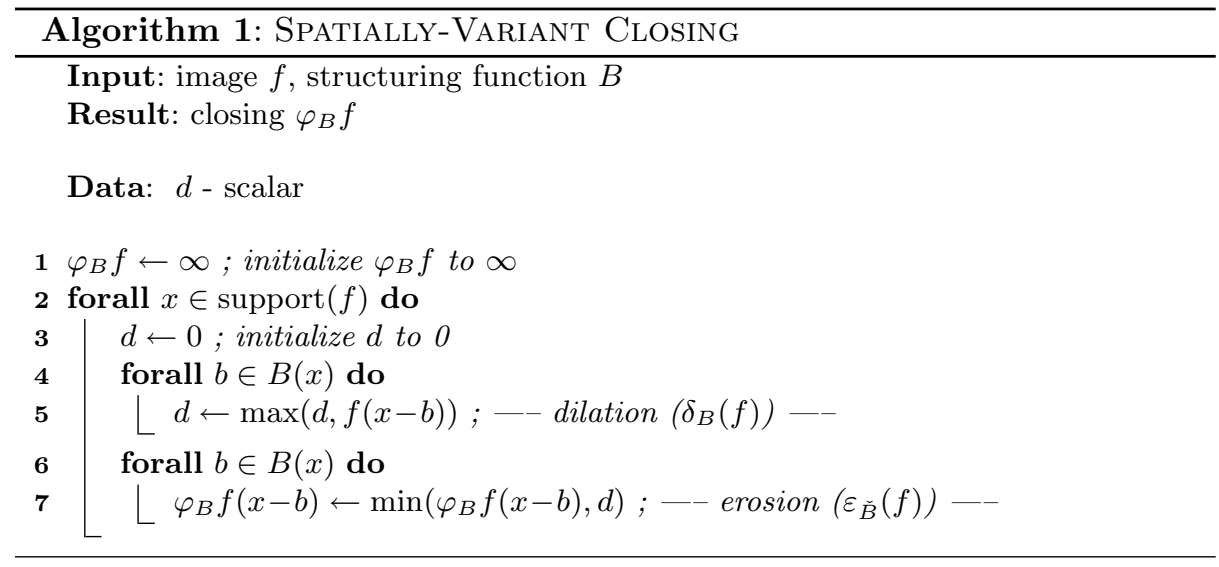

and so $H$ is only estimated, at a certain scale $\sigma$, via a convolution of $L$ by a Gaussian of parameter $\sigma$ and a discrete derivative operator [1]. Eigen-analysis of $H$ can reveal local structure information. In this paper we focus on the case $n=3$, with the three eigenvalues, $\left|\lambda_{1}\right|,\left|\lambda_{2}\right|,\left|\lambda_{3}\right|$, of $H$ in increasing order, and $\left(\mathbf{e}_{\mathbf{1}}, \mathbf{e}_{\mathbf{2}}, \mathbf{e}_{\mathbf{3}}\right)$ as their corresponding eigenvectors.

\subsection{Line detection using the Hessian in 3D}

Here we mostly follow the formulations by [11,13]. Tubular objects in 3D are indicated by features which exhibit low curvature along the main axis of the object, and strong curvature in the perpendicular plane to this axis. In Hessian eigenspace, this translates to $\left|\lambda_{3}\right| \geq\left|\lambda_{2}\right| \geq\left|\lambda_{1}\right|$, and the main axis is indicated by $\mathbf{e}_{\mathbf{1}}$ (in the case of a bright vessel). Consequently, $V=\lambda_{3}-\lambda_{2}$ is a simple tubular indicator, which is of high value in tubular structures. Frangi [13] has a more sophisticated model for computing a so-called "vesselness measure" involving multiscale analysis to estimate the probability of a voxel to belong to a blood vessel:

$$
\nu(x, \sigma)=\left\{\begin{array}{l}
0 \quad \text { if } \lambda_{2}>0 \text { or } \lambda_{3}>0, \\
\left(1-e^{\frac{-R_{A}^{2}}{2 \alpha^{2}}}\right) \cdot e^{-\frac{R_{B}^{2}}{2 \beta^{2}}} \cdot\left(1-e^{\frac{-S^{2}}{2 \gamma^{2}}}\right) \text { otherwise, }
\end{array}\right.
$$

with

$$
\begin{aligned}
& R_{A}=\frac{\left|\lambda_{2}\right|}{\left|\lambda_{3}\right|}, \\
& R_{B}=\frac{\left|\lambda_{1}\right|}{\sqrt{\left|\lambda_{2} \lambda_{3}\right|}}, \\
& S=\left\|H_{\sigma}\right\|=\sqrt{\sum_{j} \lambda_{j}^{2}},
\end{aligned}
$$

in which $R_{A}$ differentiates between plate and line like objects, $R_{B}$ describes blob-like ones, and $S$ accounts for the intensity difference between these objects and background. Parameters $\alpha, \beta$ and $\gamma$ influence the weight of the according objects. The final vesselness result is produced by its best response at different scales $\sigma$ for each voxel $x$. 


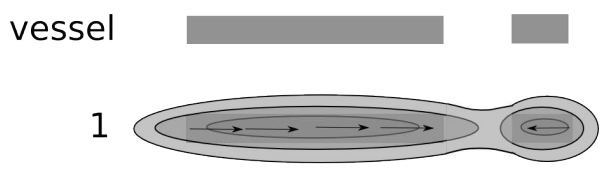

2

Fig. 4. Illustration of the directional closing. The original, noisy, broken vessel is at the top. Vessel directions are estimated from the Hessian (first step). A SV dilation and its adjunct erosion are performed in the main direction of the vessel (second step). To eliminate noise, a reconstruction from the vesselness is also performed.

Blood vessels are not strictly made of tubular structures, but also of junctions, more complex, spongious areas, and stenosis, forming double conic shapes. In general, junctions and also thin objects that are not tube-like, e.g. membranes, surfaces or double cones, can also be characterized to some degree with the Hessian analysis but ultimately will require more sophisticated analysis. There are works that have proposed Hessian-based formulations of these kind of objects $[27,28]$.

In the application part of this article, we only consider the tubular object detection problem.

\section{Application}

In this section we describe an application where combined Hessian and morphological analysis is useful. More specifically, we propose to improve Frangi's vesselness measure by morphological SV closing, allowing to reduce the noise and vessel disconnections.

\subsection{Algorithm}

The steps of the algorithm are illustrated in the Figure 4. In the first step of the algorithm, the Hessian analysis is performed at each pixel on multiple scales of the image. The vesselness as in Eq. 7 is calculated across these scales with the maximum final response for each voxel. This indicates which voxels belong to a tubular feature but fails to connect vessels portions when the noise level is too high or when vessels are too narrow. However, we observe that the orientation field is relatively robust within an appropriate choice of scales (see more discussion in Section 4.2).

In the second step, we use a family $B\left(\mathbf{e}_{\mathbf{1}}(x)\right)$ of centered segments as structuring elements oriented in the direction of $\mathbf{e}_{\mathbf{1}}$, and of fixed length for the morphological closing operation with the aim of vessel reconnection. The SE-based dilation is followed by the adjunct erosion $\delta_{B}\left(\varepsilon_{B}\right)$ as in section 2.2. In order to propagate the objects in the space, the dense orientation field in necessary. In our case, this is required only as far as the dilation can reach. At first, the 
dilation is performed only on the directional image. Then, the actual intensity image is dilated according to the directions obtained in the previous dilation. This is followed by the adjunct erosion operation. This ensures an idempotent result, which guarantees that the resulting filter obeys all morphological rules.

Following the morpho-Hessian closing - which performs like an edge-enhancing inverse diffusion filter in some ways - noise levels are generally increased. In order to filter out the noise not connected with vessels and reconnect the vessels, we perform the gray-level reconstruction [29] using the initial vesselness image as marker, and the result of the morphological closing as mask. For the simple example from the Fig. 4, the result of the grayscale reconstruction is similar to the closing.

\subsection{Results and discussion}

The described algorithm was implemented with the ITK library using the available implementations of vesselness.

The image volume is produced with MRI and is of size $256 \times 256 \times 256$ pixels. The time processing of the Hessian multiscale method is 2 minutes and of the morphological closing is 1 minute 20 seconds on a single core of a $2.5 \mathrm{GHz}$ Intel Quad-Core Xeon processor.

The considered vesselness parameters have been set to: $\alpha=\beta=0.5$ and $\gamma$ $=5$, as proposed in [13]. The $\mathrm{SE}$ is symmetric according to the origin projecting the opposite direction of the current pixel, its total length is fixed at 7 voxels. It would be also possible to modify the length of $B$ according to $\lambda_{1}$ (this might be more costly at larger scales), but experiments indicate that this is not critical.

Commonly, the lower scales are more sensitive to noise. Furthermore, in [30] it has been reported that the vesselness response curve differs not more than 3 percent between $\sigma=1$ and $\sigma=2 \sqrt{2}$ using a set of discrete values $\sigma_{i} \in 1, \sqrt{2}, 2,2 \sqrt{2}$ and that is applicable for most applications. For this filtering method, we used the scales between $\sigma=1$ and $\sigma=2 \sqrt{2}$ with three logarithmic natural incrementation steps.

The results are illustrated in Figure 5 with an application to the segmentation of brain vessels obtained with MRI angiography modality.

The original and filtered images are surface-rendered with the same threshold. The volume-rendered image (Figure 5(a)) shows that the image contains a high level of noise and disconnections that prevent using a low threshold for detecting all vessels. In Figure 5(b), it can be observed that the vesselness filters out the noise, but does not manage to reconnect the noise-corrupted vessels. However, the orientation of the first eigenvector (Figure 5(c)) remains relatively unchanged by the noise level, due to Gaussian filtering in the Hessian estimation (the turbulence-like effect of the vector flow is an artifact of a visualisation program). Thanks to these principal vessel directions and in the contrast to the vesselness filter, morpho-Hessian filter (Figure 5(d)), accomplishes reconnections of the smaller vessel parts and eliminates the noise, up to some extent.

The gray-level reconstruction eliminates all non-connected noise of the result of morpho-Hessian filter. The final segmentation is achieved by top-hat thresh- 


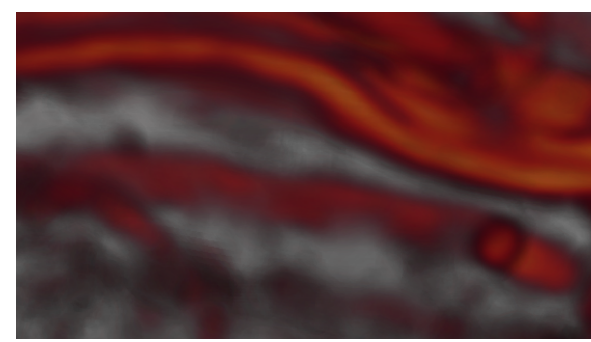

(a) Volume rendering of small arteries of the brain (in red) in MRI-TSA modality.

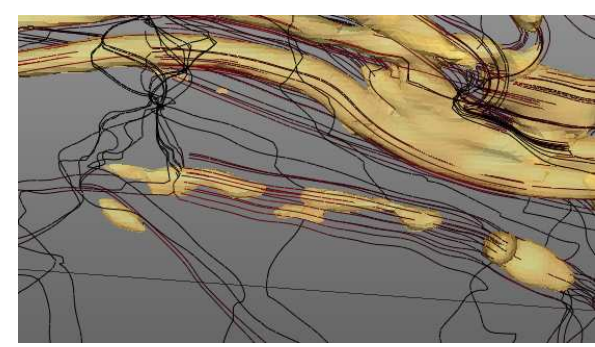

(c) Vector flow lines of the principal eigenvectors oriented in the main direction of the vessel.

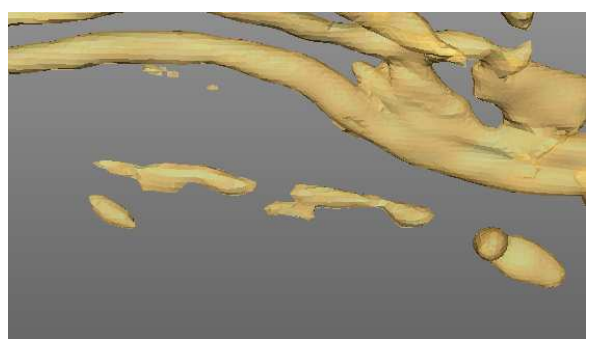

(b) Surface rendering of the vesselness response.

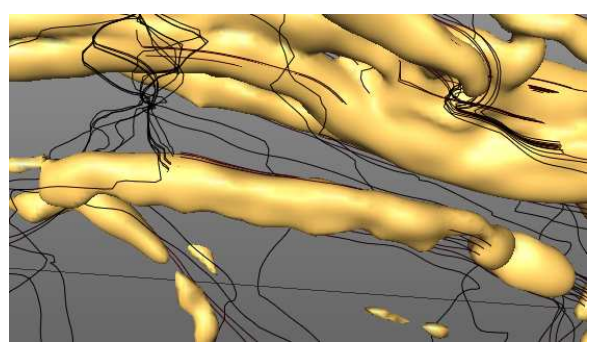

(d) Reconstructed vessel by spatiallyvariant closing.

Fig. 5. Application to the segmentation of brain vessels.

olding [1]. The threshold parameter is chosen heuristically and is the same for all methods while an automated segmentation method is under development.

\section{Conclusions}

In this article we have presented a concise theory of spatially-variant morphology with the main result being an efficient algorithm in $\mathcal{O}(M N)$ for spatially-variant openings and closings, where $N$ is the size of the image, and $M(M \ll N)$ is the size of the structuring element. While SVMM is not in itself novel, we believe that the presentation of the algorithm in this article is simple and enlightening.

As an application, we have presented a morpho-Hessian filter for 3D image filtering, in particular, enhancement and reconnection of thin, tubular objects. Directions in 3D were obtained by eigen-analysis of the Hessian, and reconnection was achieved by SV morphology. Hessian analysis is fast, multiscale and relatively robust for object direction detection, whereas orientation analysis using known MM methods would require directional sampling, which, in turn, would be prohibitive in 3D. Conversely, anisotropic diffusion is inefficient, requiring many iterations and featuring convergence issues, whereas a closing or opening converges in one iteration. 
The results obtained by applying this filter on real 3D MRI-TSA data, compared to those acquired with the vesselness function [13], underline the aptitude of the suggested routine for reconnection of the smaller vessels.

Our proposed filter also requires very few parameters, namely the range of scales in Hessian analysis and the length of the closing/opening segment for reconnection.

Overall, this approach may be seen as a productive combination of linear and non-linear techniques. Future work includes studying alternatives to the Hessian tensor for improved noise robustness and direction detection. The handling of scales with regard to the topology of the vascular tree could also be considered in further works. And, as proposed in [23], the size of the spatially-variant structuring elements could be resized according to the eigenvalues of the Hessian matrix. Lastly, a more complete application involving large-scale reconstruction of the brain vascular system, its semi- or completely automatic segmentation and classification of arteries and veins are our ultimate goals.

\section{Acknowledgments}

We wish to thank the following medical personnel from Colmar Hospital: A. Tournade, M. Musacchio, H. Oesterl, M. Lagneau, who provided medical expertise and data. This work was partially supported by grant SURF ANR-05BLAN-0071, reference NT05-2_45825.

\section{References}

1. Gonzalez, R., Woods, R.: Digital Image Processing. 3rd edn. Prentice-Hall (2007)

2. Heijmans, H.: Morphological filters for dummies. In Maragos, P., Schafer, R.W., Butt, M.A., eds.: Mathematical Morphology and its Applications to Image and Signal Processing, Atlanta, GA, proceedings for ISMM'96, Kluwer Acad. (May 1996) 127-137

3. Perona, P., Malik, J.: Scale-space and edge detection using anisotropic diffusion. IEEE Transactions on Pattern Analysis and Machine Intelligence 12(7) (1990) 629-639

4. Smith, S.M., Brady, J.M.: Susan a new approach to low level image processing. Int. J. Comput. Vision 23(1) (1997) 45-78

5. Tomasi, C., Manduchi, R.: Bilateral filtering for gray and color images. Computer Vision, 1998. Sixth International Conference on (1998) 839-846

6. Soille, P., Talbot, H.: Directional morphological filtering. IEEE Transactions on Pattern Analysis and Machine Intelligence 23(11) (2001) 1313-1329

7. Heijmans, H., Buckley, M., Talbot, H.: Path openings and closings. Journal of Mathematical Imaging and Vision 22 (2005) 107-119

8. Talbot, H., Appleton, B.: Efficient complete and incomplete paths openings and closings. Image and Vision Computing 25(4) (April 2007) 416-425

9. Zana, F., Klein, J.C.: Segmentation of vessel-like patterns using mathematical morphology and curvature evaluation. IEEE Transactions on Image Processing 10(7) (2001) 1010-1019 
10. Lindeberg, T.: Feature detection with automatic scale selection. International Journal of Computer Vision 30(2) (1998) 79-116

11. Danielsson, P.E., Lin, Q.: Efficient detection of second-degree variations in $2 \mathrm{D}$ and 3D images. Journal of Visual Communication and Image Representation 12 (2001) 255-305

12. Deguchi, K., Izumitani, T., Hontani, H.: Detection and enhancement of line structures in an image by anisotropic diffusion. Pattern Recognition Letters 23(12) (October 2002) 1399-1405

13. Frangi, A., Niessen, W., Vincken, K., Viergever, M.: Multiscale vessel enhancement filtering. In: MICCAI. Volume 1496 of LNCS. (1998) 130-137

14. Manniesing, R., Viergever, M., Niessen, W.: Vessel enhancing diffusion: A scale space representation of vessel structures. Medical Image Analysis 10(6) (2006) 815-825

15. Perona, P.: Orientation diffusions. IEEE Trans. Image Processing 7 (1998) 457-467

16. Lee, T., Talbot, H.: A fast method for detecting and matching linear features in images. In: Proc. DICTA, Brisbane, Australia (Dec. 1995) 649-654

17. Vincent, L.: Minimal path algorithms for the robust detection of linear features in images. In: Mathematical Morphology and its Applications to Image and Signal processing. Volume 12 of Computational Imaging and Vision., Amsterdam, Kluwer (June 1998) 331-338 Proceedings for ISMM'98.

18. Cohen, L., Deschamps, T.: Segmentation of 3 d tubular objects with adaptive front propagation and minimal tree extraction for $3 \mathrm{~d}$ medical imaging. Computer Methods in Biomechanics and Biomedical Engineering 10(4) (August 2007) 289305

19. Verdú-Monedero, R., Angulo, J.: Spatially-variant directional mathematical morphology operators based on a diffused average squared gradient field. In: ACIVS. Volume 5259 of LNCS., Springer (2008) 542-553

20. Tankyevych, O., Talbot, H., Dokladal, P.: Curvilinear morpho-Hessian filter. In: IEEE ISBI. (2008) 1011-1014

21. Serra, J.: Image analysis and mathematical morphology. Academic Press (1982)

22. Lerallut, R., Decencière, E., Meyer, F.: Image filtering using morphological amoebas. Image and Vision Computing 25(4) (2007) 395-404

23. Dokládal, P., Dokládalová, E.: Grey-scale morphology with spatially-variant rectangles in linear time. In: ACIVS. Volume 5259 of LNCS. (2008) 674-685

24. Charif-Chefchaouni, M., Schonfeld, D.: Spatially-variant mathematical morphology. Image Processing, 1994. Proceedings. ICIP-94., IEEE International Conference 2 (Nov 1994) 555-559 vol.2

25. Bouaynaya, N., Charif-Chefchaouni, M., Schonfeld, D.: Theoretical foundations of spatially-variant mathematical morphology part i: Binary images. IEEE Trans. Pattern Anal. Mach. Intell. 30(5) (2008) 823-836

26. Bouaynaya, N., Schonfeld, D.: Theoretical foundations of spatially-variant mathematical morphology part ii: Gray-level images. IEEE Trans. Pattern Anal. Mach. Intell. 30(5) (2008) 837-850

27. Agam, G., Armato, S.G., I., Wu, C.: Vessel tree reconstruction in thoracic ct scans with application to nodule detection. Medical Imaging, IEEE Transactions on 24(4) (April 2005) 486-499

28. Antiga, L.: Generalizing vesselness with respect to dimensionality and shape. ISC/NA-MIC Workshop on Open Science at MICCAI (8 2007)

29. Vincent, L.: Morphological grayscale reconstruction in image analysis: Applications and efficient algorithms. IEEE Transactions on Image Processing 2(2) (1993) 176201 
30. Sato, Y., Westin, C.F., Bhalerao, A., Nakajima, S., Shiraga, S., Tamura, S., Kikinis, R.: Tissue classification based on 3D local intensity structures for volume rendering. IEEE Transactions on Visualization and Computer Graphics 6(2) (2000) 160-180 